\title{
Evaluation of Fungal Activity Through In Silico Analysis of Medicinal Plants Against Exophiala Jeanselmei
}

\author{
V. Sankara Vel and A. Arunprasath* \\ Department of Botany, PSG College of Arts \& Science, Coimbatore, Tamil Nadu, India
}

* Corresponding Author email:

arunprasath@psgcas.ac.in

Article History

Received: 08 September 2020

Revised: 05 January 2021

Accepted: 25 January 2021

Published: 31 January 2021

Student(s)

- V. Sankara Vel

Academic Year: 2019-20

Course Level: Master

Course Name: M.Sc. (Botany)

Course year: Final Year

$\operatorname{Mentor}(s)$

- Dr. A. Arunprasath

\section{ABSTRA C T}

Phaeohyphomycosis is a fungal infectious disease commonly called as dermal problem which is caused by dematiaceous fungi, Exophiala jeanselmei. Chitin was the main component of fungal cell and no effective inhibitor was identified still in chitin synthase I. The protein chitin synthase I play a major role in drug metabolism as well as signal processing molecule and therefore have been targeted in the present study. The medicinal plants being a solution for several human ailments, also act as a reservoir for secondary metabolites, has taken its credit as a cure from our ancient times. The biological activity of the Myricetin was analysed using the pass online tool. The value of Probability to be active $(\mathrm{Pa})=0.241$ Probability to be inactive $(\mathrm{Pi})=0.021$. The several compounds retrieved from the plants Acalypha indica, Achyranthus aspera, Brassica niger, Cassia auriculata, Cleome gynandra, Clitoria ternatea, Ipomoea hederaceae, Leucas aspera, Mimosa pudica, Phyllanthus niruri, Ocimum basilicum, Ocimum sanctum, Tridax procumbens, Vitex negundo and Waltheria indica were analyzed for its possible significant interaction with the target protein using molecular docking studies. The compound Myricetin had Binding energy of $-7.32 \mathrm{Kcal} / \mathrm{mol}$ and formed hydrogen bonds with the residue HIS 29 showing the bond length of $1.8 \AA$ and residue THR 3 showing the bond length of 1.9 $\AA$. The future perspective of the study is to determine the stability of the protein-compound interaction through docking studies.

Keywords: Phaeohyphomycosis, Medicinal plants and Molecular docking.

\section{Introduction}

Bioinformatics is computational biology in terms of molecules and applying informatics techniques from various disciplines to understand and organize the information associated with these molecules, on a large scale [1]. Modern bioinformatics research does not necessarily require more resources than any other field of Computer Science; almost all processes can be efficiently designed and modeled on a personal computer or workstation [2]. For more than a century, vast progress has been made in hereditary qualities and molecular biology. New high-throughput exploratory methods continued to develop rapidly. The mechanization of DNA sequencing set up for the Human Genome Project in 1990 [3], which has prompted genomics and a scope of related disciplines, for example, transcriptomics (the investigation of the total 
quality articulation state), proteomics (the investigation of the full arrangement of proteins encoded by a genome), and metabolomics (the investigation of extensive metabolite profiles), here and there every one of these areas are all things considered alluded to as genomics. Numerous institutes use PC bunches to expand handling time, increment information base stockpiling and actualize quicker information putting away and recovering techniques [4]. The significant favorable circumstances of utilizing PC bunches are clear when an association requires enormous scope processing like in bioinformatics educating and research. At the point when utilized along these lines, PC groups offer: Cost productivity: the bunch method is practical for the measure of intensity and preparing speed being delivered. At present, progressed bioinformatics is amassed in a couple of exploration bases and privately owned businesses on the world that have the ability to utilize staff with profoundly particular preparing. Notwithstanding the way that bioinformatics techniques are openly available, there is obviously a hole between the creating and the industrialized world, which must be intentionally limited.

Following the development of the first algorithms in the 1980s, molecular docking became an essential tool in drug discovery [5]. In molecular docking, in light of the protein structures, a great many potential stances of association are attempted and assessed; the posture with the least energy score is anticipated as the "best match", i.e., the coupling mode. Since Kuntz and associates spearheading work [6], critical advancement has been made in mooring exploration to improve the computational speed and precision. Among them, protein-ligand docking is an especially energetic exploration region in view of its significance to structurebased medication design [7] and will be the subject of the current survey [8].

Bioinformatics was characterized as the assortment, order, stockpiling, and investigation of biochemical and biological data utilizing through in silico analysis of molecular genetics and genomics. Phaeohyphomycosis is a rare mycotic infection caused by various heterogeneous groups of black colored fungi (dematiaceous) involving the skin and subcutaneous tissue. Phaeohyphomycosis is due to an irresistible pigment called melanin which is regularly known for dermal issues brought about by dematiaceous growths, Exophiala jeanselmei. It is a crafty microorganism skilled to most regularly display phaeomycotic cyst/subcutaneous phaeohyphomycosis. Chitin a basic segment of parasitic cell divider is one of a kind to contagious realm and interim, no practical inhibitor was so far distinguished for chitin synthase, however the melanin shade present in the cell mass of Exophiala jeanselmei causes the destructiveness reason for the microorganism. In later phases of this illness goes to the mind and causes the demise [9]. The most widely recognized etiological operators of subcutaneous Phaeohyphomycosis are E. jeanselmi followed by E. dermatitidis [10]. The genus Exophiala is generally right in the earth and may cause contaminations in both immune compromised and once in a while, in immunocompetent people. E. jeanselmi ordinarily causes gentle cutaneous and subcutaneous diseases which are often limited and singular (phaeohypomycotic blister) [11]. Indeed, even in seriously immune suppressed patients Exophiala contamination regularly will in general remain restricted [12]. Ajello recorded 71 types of dematiaceous organisms from 39 genera which have been found to cause phaeohyphomycosis in people and in lower creatures [13]. There have been two distributed reports of Phaeohyphomycosis brought about by Exophiala jeanselmei in a household feline [14] and in Australia Phaeohyphomycosis brought about by Exophiala spinifera in two felines [15]. There are various cases in the writing in which the analysis was founded exclusively on histopathology, which, albeit trademark, gives in sign with regards to the character of the organism included [16]. Phaeohyphomycosis is a less frequent, progressively disfiguring and sometimes fatal infection. Although the diseases will be quite opposite, their drug therapy has become a common feature for both infections [17]. Exophiala jeanselmei is clinically deflected as an uncommon operator of subcutaneous. Our results will be discussed about its extraordinary clinical introduction and etiological operator, Exophiala jeanselmei. The patient recouped totally after treatment with Ketoconazole [18].

Therapeutic plants were made use of customary medication rehearses since ancient occasions. Plants incorporate many compounds for size comprises guard against parasites, infections, and herbivorous warmblooded creatures. Various photochemical with potential or set up organic action had been recognized. 
Restorative plants are the "spine" of customary medication, which implies more than 3.3 billion people in the less evolved nations use therapeutic plants all the time [19]. There are about 2000 ethnic gatherings on the planet, and pretty much every gathering their own conventional clinical information and encounters [20]. Recent screenings of most of the medicinal plant extracts with antibacterial, anticandidal and antidermatophytic efficacy shown better because of those active compounds which could be isolated and has been able to identify promising compounds that might represent future solutions in critical areas of human health $[21,22]$. Therapeutic plant compounds have just been utilized to effectively treat various viral illnesses. Thus, we screened a therapeutic plant information base containing 32,297 potential enemies of viral phytochemicals and chosen the main nine hits that may repress SARS-CoV-2 3CLpro action also, thus infection replication [23].

As indicated by WHO, the vast majority of the developing and developed nations accept on home grown items for its therapeutic accessibility, in light of this philosophy the accompanying medicinal plants are utilized for the treatment of Phaeohyphomycosis. The aim of this study is to cure this dermal disease by knockout the Melanin pigment which is the main virulent factor by Molecular docking studies (Acalypha indica, Achyranthus aspera, Brassica niger, Cassia auriculata, Cleome gynandra, Clitoria ternatea, Ipomoea hederaceae, Lencas aspera, Mimosa pudica, Phyllanthus niruri, Ocimum basilicum, Ocimum sanctum, Tridax procumbens, Vitex negundo and Waltheria indica)

\section{Materials and Methods}

\subsection{Pdb, Pubchem \& Pass Online}

The 3D protein structure for Chitin syntheses (Chs) of Exophiala jeanselmei is recovered from the Protein Data Bank database (PDB ID: 2MPK). Dynamic web page region was anticipated utilizing LigSite online apparatus. The concoction mixes from the referenced plants are recovered from the PubChem database. The PASS ONLINE predicts 4130 types of biological activities, for which the difference between probabilities will be active $(\mathrm{Pa})$ and probabilities will be inactive $(\mathrm{Pi})$ was calculated. The $\mathrm{Pa}-\mathrm{Pi}$ values for activities randomly selected from the total list of predicted biological activities will be used as independent regression variables are perused.

\subsection{Drugability}

Lipinski rule of 5 helps in distinguishing between drug like and non-drug like molecules. It predicts high probability of success or failure due to drug likeness for molecules complying with 2 or more of the following rules [22 \& 24]. Molecular mass less than 500 Dalton

$>\quad$ High lipophilicity (expressed as LogP less than 5)

$>\quad$ Less than 5 hydrogen bond donors

$>$ Less than 10 hydrogen bond acceptors

$>\quad$ Molar refractivity must between 40-130

\subsection{Molecular Docking Study}

MGL tools with AutoGrid4 and AutoDock4 [25] will be used to set up and to perform blind docking calculations between the Ligands and Protein. Crystallized 3-dimensional structure was obtained from the Protein Data Bank (PDB). Receptor (protein) and ligand (complex) files were prepared using Auto Dock Tools. The protein was enclosed in a box with grid points in $\mathrm{x}, \mathrm{y}$ and $\mathrm{z}$ directions and a grid spacing of $0.375 \AA$. The center of the grid set to $-6.516,30.278$ and $-1.951 \AA$. Lamarckian genetic algorithms, as implemented in Auto Dock, were employed to perform docking calculations. All other guidelines are default settings. For every individual the docking cases, the lowest energy docked conformation, according to the Auto Dock scoring function and number hydrogen bonds was selected as the binding mode. The output from Auto Dock was rendered with PyMol [26]. 


\subsection{PyMOL}

PyMOL is one of a few open source visualization tools which are used in a structural biology. A part of the software's name refers to the fact that it extends, and is extensible by the python programming language. All the bindings are visualized by using the Structure Visualing tool Pymol viewer, the interaction between the chemical compounds and target protein.

\section{Results and discussion}

\subsection{PDB, PUBCHEM \& Pass Online}

In a progression of novel InhA inhibitors was recognized through a virtual screening technique. The creators utilized a multistage approach, incorporating pharmacophore demonstrating and atomic docking [27]. Most of the plants tried are a significant wellspring of hostile to parasitic aggravates that may give sustainable wellsprings of helpful antifungal medications against dermatophytic diseases in people [28]. Plants produce a combination of restorative portions as helper metabolites, for instance, phenolic blends, principal oils, tannins, terpenes, etc that can stifle microorganism advancement and are for the most part surveyed for its sensibility and reasonableness [29] various components add to anti-toxin opposition remembering abuse and abuse of anti-microbials for people, creatures and horticulture; patient's interest for and receipt of anti-infection agents when they needn't bother with them; and inability to complete an anti-microbial solution. Accordingly, the utilization of Ayurveda drugs has expanded now days [30]. The aeronautical pieces of $C$. auriculata L. shows higher antibacterial and antifungal action against bacterial and contagious microbes, for example, Bacillus subtilis, Staphylococcus aureus, Psendomonas aeruginosa, Escherichia coli, Candida albicans and Aspergillus niger [31].

Table 1: Structure of Protein

\begin{tabular}{|c|c|c|c|}
\hline ORGANISM NAME & PROTEIN NAME \& ID & STRUCTURE & $\begin{array}{c}\text { RESIDUES } \\
\text { COUNT }\end{array}$ \\
\hline Exophiala jeanselmei & Chitin Synthase I \& 2MPK & & \\
\hline
\end{tabular}

The 3D structure of a protein (Chitin synthase I) was recovered from the Protein Data Bank (PDB) and the information was mentioned (Table 1). It was visualized by utilizing the visualizing tool pymol. The three dimensional structure of the protein was distinguished utilizing Ligsite online device. The 3D structures of the phytochemical compounds of the plants chosen in the current investigation were retrieved from Pubchem Chemical database. The compounds which are segregated and revealed from earlier mentioned restorative plants were organized (Table 2) along with their molecular weight, molecular formula and pubchem ID, where pubchem is the database for getting small molecules. The biological activity of the Myricetin was broke down utilizing the pass online device which predicts the pharmacological and biochemical properties. The estimation of Probability to be dynamic $(\mathrm{Pa})=0.241$ Probability to be idle $(\mathrm{Pi})$ $=0.021$. The properties anticipated for each plant compounds were recorded (Table 3). 
V. Sankara Vel and A. Arunprasath, Adv. J. Grad. Res.; Vol. 9, Issue 1, pp: 81-95, January 2021

Table 2: List of compounds isolated from medicinal plants

\begin{tabular}{|c|c|c|c|c|}
\hline S.No & Compound Name & Pubchem ID & $\begin{array}{c}\text { Molecular } \\
\text { formula }\end{array}$ & Molecular Weight \\
\hline 1. & Myricetin & 5281672 & $\mathrm{C}_{15} \mathrm{H}_{10} \mathrm{O}_{8}$ & $318.237 \mathrm{~g} / \mathrm{mol}$ \\
\hline 2. & Quercetin & 5280343 & $\mathrm{C}_{15} \mathrm{H}_{10} \mathrm{O}_{7}$ & $302.238 \mathrm{~g} / \mathrm{mol}$ \\
\hline 3. & Daucosterol & 5742590 & $\mathrm{C}_{35} \mathrm{H}_{60} \mathrm{O}_{6}$ & $576.859 \mathrm{~g} / \mathrm{mol}$ \\
\hline 4. & Baicalein & 5281605 & $\mathrm{C}_{15} \mathrm{H}_{10} \mathrm{O}_{5}$ & $270.24 \mathrm{~g} / \mathrm{mol}$ \\
\hline 5. & Cosmosin & 5280704 & $\mathrm{C}_{21} \mathrm{H}_{20} \mathrm{O}_{10}$ & $432.381 \mathrm{~g} / \mathrm{mol}$ \\
\hline 6. & Kaempferol & 5280863 & $\mathrm{C}_{15} \mathrm{H}_{10} \mathrm{O}_{6}$ & $286.239 \mathrm{~g} / \mathrm{mol}$ \\
\hline 7. & Robinetin & 5281692 & $\mathrm{C}_{15} \mathrm{H}_{10} \mathrm{O}_{7}$ & $302.238 \mathrm{~g} / \mathrm{mol}$ \\
\hline 8. & Luteolin & 5280445 & $\mathrm{C}_{15} \mathrm{H}_{10} \mathrm{O}_{6}$ & $286.239 \mathrm{~g} / \mathrm{mol}$ \\
\hline 9. & Acacetin & 5280442 & $\mathrm{C}_{16} \mathrm{H}_{12} \mathrm{O}_{5}$ & $284.267 \mathrm{~g} / \mathrm{mol}$ \\
\hline 10. & Catechin & 9064 & $\mathrm{C}_{15} \mathrm{H}_{14} \mathrm{O}_{6}$ & $290.271 \mathrm{~g} / \mathrm{mol}$ \\
\hline 11. & Epicatechin & 72276 & $\mathrm{C}_{15} \mathrm{H}_{10} \mathrm{O}_{6}$ & $290.271 \mathrm{~g} / \mathrm{mol}$ \\
\hline 12. & Isovitexin & 162350 & $\mathrm{C}_{21} \mathrm{H}_{20} \mathrm{O}_{10}$ & $432.381 \mathrm{~g} / \mathrm{mol}$ \\
\hline 13. & +)- Gallacatechin & 65084 & $\mathrm{C}_{15} \mathrm{H}_{14} \mathrm{O}_{7}$ & $306.27 \mathrm{~g} / \mathrm{mol}$ \\
\hline 14. & 3-o-Methyl-D-Glucose & 8973 & $\mathrm{C}_{7} \mathrm{H}_{14} \mathrm{O}_{6}$ & $194.183 \mathrm{~g} / \mathrm{mol}$ \\
\hline 15. & Chanoclavine & 5281381 & $\mathrm{C}_{15} \mathrm{H}_{20} \mathrm{~N}_{2} \mathrm{O}$ & $256.349 \mathrm{~g} / \mathrm{mol}$ \\
\hline 16. & Adenosine & 60961 & $\mathrm{C}_{10} \mathrm{H}_{13} \mathrm{~N}_{5} \mathrm{O}_{4}$ & $267.245 \mathrm{~g} / \mathrm{mol}$ \\
\hline 17. & Naringenin & 932 & $\mathrm{C}_{15} \mathrm{H}_{15} \mathrm{O}_{2}$ & $272.256 \mathrm{~g} / \mathrm{mol}$ \\
\hline 18. & Daidzein & 5281708 & $\mathrm{C}_{15} \mathrm{H}_{10} \mathrm{O}_{4}$ & $254.241 \mathrm{~g} / \mathrm{mol}$ \\
\hline 19. & Penninclavine & 115247 & $\mathrm{C}_{16} \mathrm{H}_{18} \mathrm{~N}_{2} \mathrm{O}_{2}$ & $270.332 \mathrm{~g} / \mathrm{mol}$ \\
\hline 20. & Isopenniclavine & 12311156 & $\mathrm{C}_{16} \mathrm{H}_{18} \mathrm{~N}_{2} \mathrm{O}_{2}$ & $270.332 \mathrm{~g} / \mathrm{mol}$ \\
\hline 21. & Esculetin & 5281416 & $\mathrm{C}_{9} \mathrm{H}_{6} \mathrm{O}_{4}$ & $178.143 \mathrm{~g} / \mathrm{mol}$ \\
\hline 22. & Ciprofloxacin & 2764 & $\mathrm{C}_{17} \mathrm{H}_{18} \mathrm{~N}_{3} \mathrm{O}_{3}$ & $331.347 \mathrm{~g} / \mathrm{mol}$ \\
\hline 23. & Lyserol & 14987 & $\mathrm{C}_{16} \mathrm{H}_{18} \mathrm{~N}_{2} \mathrm{O}$ & $254.333 \mathrm{~g} / \mathrm{mol}$ \\
\hline 24. & Pueranin & 5281807 & $\mathrm{C}_{21} \mathrm{H}_{20} \mathrm{O}_{9}$ & $416.382 \mathrm{~g} / \mathrm{mol}$ \\
\hline 25. & 6-Hydroxy lavone & 72279 & $\mathrm{C}_{15} \mathrm{H}_{10} \mathrm{O}_{3}$ & $238.242 \mathrm{~g} / \mathrm{mol}$ \\
\hline 26. & Teucladiol & 1604618 & $\mathrm{C}_{15} \mathrm{H}_{26} \mathrm{O}_{2}$ & $238.371 \mathrm{~g} / \mathrm{mol}$ \\
\hline 27. & Ethyl caffetate & 5317238 & $\mathrm{C}_{11} \mathrm{H}_{12} \mathrm{O}_{6}$ & $208.213 \mathrm{~g} / \mathrm{mol}$ \\
\hline 28. & Buetin & 5281222 & $\mathrm{C}_{15} \mathrm{H}_{12} \mathrm{O}_{5}$ & $272.256 \mathrm{~g} / \mathrm{mol}$ \\
\hline 29. & 20-hydroxyecdysone & 5459840 & $\mathrm{C}_{22} \mathrm{H}_{44} \mathrm{O}_{7}$ & $480.642 \mathrm{~g} / \mathrm{mol}$ \\
\hline 30. & Rubiadin & 5124062 & $\mathrm{C}_{15} \mathrm{H}_{10} \mathrm{O}_{6}$ & $473.943 \mathrm{~g} / \mathrm{mol}$ \\
\hline
\end{tabular}




\begin{tabular}{|c|c|c|c|c|}
\hline 31. & Mimosic acid & 190359 & $\mathrm{C}_{7} \mathrm{H}_{2} \mathrm{NO}_{4}$ & $169.136 \mathrm{~g} / \mathrm{mol}$ \\
\hline 32. & Octadecatrienoic acid & 5739740 & $\mathrm{C}_{28} \mathrm{H}_{34} \mathrm{O}_{4}$ & $434.576 \mathrm{~g} / \mathrm{mol}$ \\
\hline 33. & Tetracaine & 5411 & $\mathrm{C}_{15} \mathrm{H}_{24} \mathrm{~N}_{2} \mathrm{O}_{2}$ & $264.369 \mathrm{~g} / \mathrm{mol}$ \\
\hline 34. & Chicanine & 5336043 & $\mathrm{C}_{20} \mathrm{H}_{22} \mathrm{O}_{5}$ & $342.391 \mathrm{~g} / \mathrm{mol}$ \\
\hline 35. & Saupirin & 181128 & $\mathrm{C}_{19} \mathrm{H}_{22} \mathrm{O}_{6}$ & $346.379 \mathrm{~g} / \mathrm{mol}$ \\
\hline 36. & Elymoclavine & 440904 & $\mathrm{C}_{16} \mathrm{H}_{18} \mathrm{~N}_{2} \mathrm{O}$ & $254.333 \mathrm{~g} / \mathrm{mol}$ \\
\hline 37. & Tyrosine & 6057 & $\mathrm{C}_{9} \mathrm{H}_{11} \mathrm{NO}_{3}$ & $181.191 \mathrm{~g} / \mathrm{mol}$ \\
\hline 38. & p-coumaric acid & 637542 & $\mathrm{C}_{9} \mathrm{H}_{8} \mathrm{O}_{3}$ & $164.16 \mathrm{~g} / \mathrm{mol}$ \\
\hline 39. & Nectandrin-b & 156517 & $\mathrm{C}_{20} \mathrm{H}_{24} \mathrm{O}_{5}$ & $344.407 \mathrm{~g} / \mathrm{mol}$ \\
\hline 40. & Caffeic acid & 689043 & $\mathrm{C}_{9} \mathrm{H}_{8} \mathrm{O}_{4}$ & $180.159 \mathrm{~g} / \mathrm{mol}$ \\
\hline 41. & Linoleic acid & 5280450 & $\mathrm{C}_{18} \mathrm{H}_{32} \mathrm{O}_{2}$ & $280.452 \mathrm{~g} / \mathrm{mol}$ \\
\hline 42. & Negundin A & 10043572 & $\mathrm{C}_{20} \mathrm{H}_{16} \mathrm{O}_{6}$ & $352.342 \mathrm{~g} / \mathrm{mol}$ \\
\hline 43. & Buchariol & 101009028 & $\mathrm{C}_{15} \mathrm{H}_{26} \mathrm{O}_{2}$ & $238.371 \mathrm{~g} / \mathrm{mol}$ \\
\hline 44. & Phyllnirurin & 179963 & $\mathrm{C}_{20} \mathrm{H}_{22} \mathrm{O}_{5}$ & $342.391 \mathrm{~g} / \mathrm{mol}$ \\
\hline 45. & Waltherine A & 100978900 & $\mathrm{C}_{31} \mathrm{H}_{42} \mathrm{~N}_{4} \mathrm{O}_{4}$ & $534.701 \mathrm{~g} / \mathrm{mol}$ \\
\hline 46. & Steroid O sulfate & 439761 & $\mathrm{C}_{18} \mathrm{H}_{24} \mathrm{O}_{4} \mathrm{~S}$ & $336.446 \mathrm{~g} / \mathrm{mol}$ \\
\hline 47. & 9,12-octadecadiynoic acid & 1931 & $\mathrm{C}_{18} \mathrm{H}_{28} \mathrm{O}_{2}$ & $276.42 \mathrm{~g} / \mathrm{mol}$ \\
\hline 48. & Silymarin & 5213 & $\mathrm{C}_{25} \mathrm{H}_{22} \mathrm{O}_{10}$ & $482.441 \mathrm{~g} / \mathrm{mol}$ \\
\hline 49. & Oleic acid & 445639 & $\mathrm{C}_{18} \mathrm{H}_{34} \mathrm{O}_{2}$ & $282.468 \mathrm{~g} / \mathrm{mol}$ \\
\hline 50. & Waltherine $\mathrm{C}$ & 100994181 & $\mathrm{C}_{30} \mathrm{H}_{37} \mathrm{~N}_{5} \mathrm{O}_{4}$ & $531.657 \mathrm{~g} / \mathrm{mol}$ \\
\hline 51. & Phyllanthin & 358901 & $\mathrm{C}_{24} \mathrm{H}_{34} \mathrm{O}_{8}$ & $418.53 \mathrm{~g} / \mathrm{mol}$ \\
\hline 52. & 9-Octadecenoic acid & 965 & $\mathrm{C}_{18} \mathrm{H}_{34} \mathrm{O}_{2}$ & $282.468 \mathrm{~g} / \mathrm{mol}$ \\
\hline 53. & Viteagnuside A & 38362716 & $\mathrm{C}_{26} \mathrm{H}_{42} \mathrm{O}_{9}$ & $498.613 \mathrm{~g} / \mathrm{mol}$ \\
\hline 54. & Niranthin & 13989915 & $\mathrm{C}_{24} \mathrm{H}_{32} \mathrm{O}_{7}$ & $432.513 \mathrm{~g} / \mathrm{mol}$ \\
\hline 55. & Bufadienolide & 46173848 & $\mathrm{C}_{24} \mathrm{H}_{34} \mathrm{O}_{2}$ & $354.534 \mathrm{~g} / \mathrm{mol}$ \\
\hline 56. & Palmitic acid & 985 & $\mathrm{C}_{16} \mathrm{H}_{32} \mathrm{O}_{2}$ & $256.43 \mathrm{~g} / \mathrm{mol}$ \\
\hline 57. & Adouetine $\mathrm{X}$ & 5281577 & $\mathrm{C}_{28} \mathrm{H}_{44} \mathrm{~N}_{4} \mathrm{O}_{4}$ & $500.684 \mathrm{~g} / \mathrm{mol}$ \\
\hline 58. & Lignans & 443013 & $\mathrm{C}_{22} \mathrm{H}_{22} \mathrm{O}_{8}$ & $414.41 \mathrm{~g} / \mathrm{mol}$ \\
\hline 59. & Oleanolic acid & 10494 & $\mathrm{C}_{30} \mathrm{H}_{48} \mathrm{O}_{3}$ & $456.711 \mathrm{~g} / \mathrm{mol}$ \\
\hline 60. & Gledigenin 1 & 45483610 & $\mathrm{C}_{30} \mathrm{H}_{48} \mathrm{O}_{3}$ & $456.711 \mathrm{~g} / \mathrm{mol}$ \\
\hline
\end{tabular}


V. Sankara Vel and A. Arunprasath, Adv. J. Grad. Res.; Vol. 9, Issue 1, pp: 81-95, January 2021

Table 3: Prediction of pharmacological activity for plant compounds

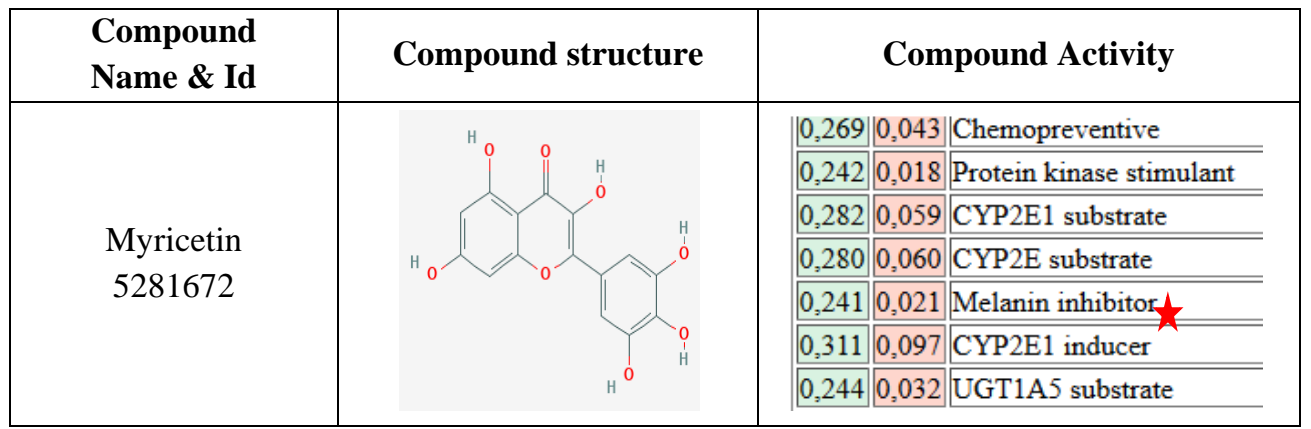

The Symbol indicates the main activity of the compound for the disease.

\subsection{Drugability}

The compounds were first oppressed for dissecting Absorption, Distribution, Metabolism and Excretion properties. Earlier ADME profiling of little atoms are noteworthy committed to the field of medication revelation by having sway on cost, work request and length [32]. Computational estimation of the docked phytochemical's medication likeliness was found based on limits set by "Lipinski's Rule of Five" through Drug examine apparatus at Molinspiration server. The subjective appraisals of ingestion, statement, digestion, discharge and poisonousness profile of these hits were anticipated basically by utilizing ADMET sar worker [33].

In the current investigation, Lipinski's standard of five in any case called Pfizer's standard of five or general guideline to assess tranquilize similarity shows the accompanying properties like atomic weight, octanol/water parcel coefficient, hydrogen bond giver and acceptor. After the filtration involving through Lipinski rule totally 39 out of 60 bioactive compounds from medicinal plants. Since the standard has a cutoff point in products of five, the name has been given as rule of five. Aside from the above properties, extra boundaries, such as, surface territory in square Armstrong (polar surface region, PSA); cerebrum/blood hindrance and level of human oral assimilation were additionally anticipated (Table 4).

Table 4: ADME toxicity for plant molecules

\begin{tabular}{|c|c|c|c|c|c|}
\hline Molecular ID & $\begin{array}{c}\text { Molecular } \\
\text { weight }\end{array}$ & $\begin{array}{c}\text { Donor Hydrogen } \\
\text { Bonds }\end{array}$ & $\begin{array}{c}\text { Acceptor } \\
\text { Hydrogen Bonds }\end{array}$ & $\begin{array}{c}\text { High lipophilicity } \\
\text { (Log P) }\end{array}$ & $\begin{array}{c}\text { Molar } \\
\text { refractivity }\end{array}$ \\
\hline Normal range & $\mathbf{5 0 0}$ & $\mathbf{5}$ & $\mathbf{1 0}$ & $\mathbf{5}$ & $\mathbf{4 0 - 1 3 0}$ \\
\hline 8973 & 194 & 4 & 6 & -2.72 & 41.96 \\
\hline 5411 & 264 & 1 & 2 & 2.89 & 80.43 \\
\hline 5280704 & 432 & 5 & 10 & -0.10 & 103.54 \\
\hline 5280863 & 286 & 4 & 6 & 0.64 & 62.82 \\
\hline 5281672 & 318 & 5 & 8 & 1.71 & 75.715 \\
\hline 5280343 & 302 & 5 & 7 & 0.52 & 64.36 \\
\hline 637542 & 164 & 2 & 3 & 0.15 & 40.19 \\
\hline 124062 & 254 & 2 & 4 & 1.66 & 61.13 \\
\hline 181128 & 346 & 2 & 6 & 2.65 & 87.76 \\
\hline
\end{tabular}




\begin{tabular}{|c|c|c|c|c|c|}
\hline 6057 & 181 & 3 & 3 & 0.56 & 44.23 \\
\hline 932 & 272 & 3 & 5 & 1.17 & 64.16 \\
\hline 2764 & 331 & 2 & 3 & 1.49 & 81.89 \\
\hline 9064 & 290 & 5 & 6 & 1.09 & 68.13 \\
\hline 14987 & 254 & 2 & 1 & 2.35 & 74.53 \\
\hline 60961 & 267 & 5 & 5 & -2.45 & 55.22 \\
\hline 65084 & 306 & 6 & 7 & 0.79 & 69.01 \\
\hline 72276 & 290 & 5 & 6 & 1.09 & 68.34 \\
\hline 72279 & 238 & 1 & 3 & 1.8 & 58.81 \\
\hline 94477 & 154 & 3 & 2 & 0.55 & 40.135 \\
\hline 115247 & 270 & 3 & 2 & 2.06 & 75.35 \\
\hline 190359 & 169 & 2 & 4 & -0.9 & 44.862 \\
\hline 440904 & 254 & 2 & 1 & 2.35 & 74.53 \\
\hline 162350 & 432 & 6 & 10 & 1.63 & 97.03 \\
\hline 5280442 & 284 & 2 & 5 & 1.65 & 67.17 \\
\hline 5280445 & 286 & 4 & 6 & 0.83 & 63.34 \\
\hline 5281222 & 272 & 4 & 5 & 1.19 & 64.86 \\
\hline 5281381 & 256 & 3 & 1 & 2.67 & 76.63 \\
\hline 5281416 & 178 & 2 & 4 & 0.45 & 46.6 \\
\hline 5281605 & 270 & 3 & 5 & 1.42 & 61.24 \\
\hline 5281692 & 302 & 5 & 7 & 0.52 & 64.36 \\
\hline 5281708 & 254 & 2 & 4 & 1.24 & 61.06 \\
\hline 5281807 & 416 & 6 & 9 & 1.74 & 96.34 \\
\hline 5317238 & 208 & 2 & 4 & 1.31 & 51.03 \\
\hline 5459840 & 480 & 4 & 7 & 5.6 & 141.7 \\
\hline 5742590 & 312 & 5 & 6 & -0.05 & 77.62 \\
\hline 57397401 & 434 & 0 & 4 & 5.77 & 130.09 \\
\hline 16046185 & 238 & 1 & 2 & 3.778 & 78.84 \\
\hline 12311156 & 270 & 3 & 2 & 2.06 & 75.6 \\
\hline 53360432 & 342 & 1 & 5 & 3.468 & 91.73 \\
\hline
\end{tabular}




\subsection{Molecular Docking Study}

Molecular docking and pharmacology studies are beneficially involved to screen the anti-inflammatory constituents of Cinnamomum cassia twigs, with a total of 69 bioactive compounds found to have potential drug-like properties. These findings will facilitate the development of bioactive compounds from C. cassia twigs for the treatment of inflammatory disorders [34]. Senna alata leaves are generally antifungal specialists they were exposed to in silico Molecular docking studies to locate their antifungal activities. As per Glide docking score selective compounds were exposed for drug-likeliness (ADME/T) investigation to foresee their conceivable potential to be used as a normally determined antifungal agent. As finalizing the results, the anthraquinones are promising applicants as an antifungal agent [35]. The results of docking studies 29 compounds were recorded (Table 5). The compound Myricetin had Binding energy of $-7.32 \mathrm{Kcal} / \mathrm{mol}$ and formed hydrogen bonds with the residue HIS 29 showing the bond length of $1.8 \AA$ and residue THR 3 showing the bond length of $1.9 \AA$. The compound Quercetin had Binding energy of $-6.82 \mathrm{Kcal} / \mathrm{mol}$ and formed hydrogen bonds with the residue ALA 26 (H-O), ASP 6 (H-O), HIS 29 (O-H) showing the bond length of 2.2, 1.7, $1.8 \AA$. The compound Daucosterol had Binding energy of $-6.64 \mathrm{Kcal} / \mathrm{mol}$ and formed hydrogen bonds with the residue HIS-66 showing the bond length of $1.6 \AA$. The compound Baicalein had Binding energy of $-6.62 \mathrm{Kcal} / \mathrm{mol}$ and formed hydrogen bonds with the residue HIS 29 and THR 3 showing the bond length of 2.2 and $2.5 \AA$. The compound Cosmosin with binding energy of $-6.58 \mathrm{Kcal} / \mathrm{mol}$ and formed hydrogen bonds with the residue ASP 32 and ASP 6 showing the bond length of 1.9 and $1.7 \AA$. Further, the interactions of plant compounds with the target would compare to the presently available drug molecule, to study its potency. As well as the simulation studies would provide an insight about the stability of protein-compound complex. The plant compounds indicating collaboration with the protein are arranged with the Binding energy. The plant compounds and the information of their hydrogen bond, collaborating deposits and individual bond lengths were organized independently (Table 6). Utilizing Pymol tool, the cooperation between the synthetic mixes and target protein are visualized and the communication of the Compound Myricetin was appeared in the figure 1.

Table 5: Docking result for plant molecules

\begin{tabular}{|c|c|c|c|}
\hline S.No & Plant name & Compounds & Binding energy \\
\hline 1. & Achyranthus aspera & 20-hydroxyecdysone (5459840) & -4.74 \\
\hline 2. & Brassica niger & Tetracaine (5411) & -4.25 \\
\hline 3. & Cassia auriculata & $\begin{array}{c}\text { Myricetin (5281672) } \\
\text { Naringenin (932) } \\
\text { Quercetin (5280343) } \\
\text { Rubiadin (124062) }\end{array}$ & $\begin{array}{l}-7.32 \\
-5.17 \\
-6.82 \\
-4.67\end{array}$ \\
\hline 4. & Cleome gynandra & $\begin{array}{l}\text { Daucosterol }(5742590) \\
\text { Teucladiol(16046185) }\end{array}$ & $\begin{array}{l}-6.64 \\
-4.81\end{array}$ \\
\hline 5. & Clitoria ternatea & $\begin{array}{c}\text { Adenosine (60961) } \\
\text { p-coumaric acid (637542) }\end{array}$ & $\begin{array}{l}-5.53 \\
-4.13\end{array}$ \\
\hline 6. & Ipomoea hederaceae & $\begin{array}{c}\text { Chanoclavine (5281381) } \\
\text { Penniclavine }(115247) \\
\text { Isopenniclavine }(12311156) \\
\text { Lysergol }(14987) \\
\text { Ethyl Caffeate }(5317238) \\
\text { Elymoclavine }(440904)\end{array}$ & $\begin{array}{l}-5.65 \\
-4.93 \\
-4.93 \\
-4.87 \\
-4.78 \\
-4.18\end{array}$ \\
\hline 7. & Leucas aspera & $\begin{array}{c}\text { Catechin (9064) } \\
\text { Acacetin (5280442) } \\
\text { Chicanine (53360432) }\end{array}$ & $\begin{array}{l}-5.76 \\
-5.91 \\
-4.22\end{array}$ \\
\hline 8. & Mimosa pudica & 6-hydroxy flavones (72279) & -4.86 \\
\hline
\end{tabular}


Evaluation of Fungal Activity Through In Silico Analysis of Medicinal Plants Against Exophiala Jeanselmei

\begin{tabular}{|c|c|c|c|}
\hline & & $\begin{array}{c}\text { Isovitexin (162350) } \\
\text { Mimosinamine (94477) } \\
\text { Mimosinic acid (190359) } \\
\text { Tyrosine (6057) }\end{array}$ & $\begin{array}{l}-5.73 \\
-5.00 \\
-4.66 \\
-4.18\end{array}$ \\
\hline 9. & Ocimum basilicum & $\begin{array}{c}\text { 3-o-methyl-d-glucose (8973) } \\
\text { Cosmosin (5280704) } \\
\text { Kaempferol (5280863) }\end{array}$ & $\begin{array}{l}-5.67 \\
-6.58 \\
-6.43\end{array}$ \\
\hline 10. & Tridax procumbens & $\begin{array}{c}\text { (+)- Gallacatechin (65084) } \\
\text { Daidzein }(5281708) \\
\text { Butein }(5281222) \\
\text { Robinetin }(5281692) \\
\text { Luteolin }(5280445) \\
\text { Baicalein }(5281605) \\
\text { Ciprofloxacin }(2764) \\
\text { Esculetin }(5281416) \\
\text { Puerarin }(5281807)\end{array}$ & $\begin{array}{l}-5.67 \\
-5.1 \\
-4.74 \\
-6.4 \\
-6.29 \\
-6.62 \\
-4.89 \\
-4.93 \\
-4.86\end{array}$ \\
\hline 11. & Vitex negundo & $\begin{array}{c}\text { Octadecatrienoic acid (57397401) } \\
\text { Saupirin }(181128)\end{array}$ & $\begin{array}{l}-4.46 \\
-4.21\end{array}$ \\
\hline 12. & Waltheria indica & Epicatechin (72276) & -5.76 \\
\hline
\end{tabular}

Table 6: Interaction of plant compounds with $2 M P K$

\begin{tabular}{|c|c|c|c|c|c|}
\hline S.No & $\begin{array}{l}\text { Name of the ligand / PubChem } \\
\text { ID }\end{array}$ & Residues Interaction & Bond length & $\begin{array}{l}\text { No of } \\
\text { Bonds }\end{array}$ & Binding energy \\
\hline \multirow{2}{*}{1.} & \multirow{2}{*}{ Myricetin/5281672 } & HIS - 29 (H-O) & 1.7 & \multirow{2}{*}{2} & \multirow{2}{*}{-7.32} \\
\hline & & THR - $3(\mathrm{H}-\mathrm{O})$ & 2.1 & & \\
\hline \multirow{3}{*}{2.} & \multirow{3}{*}{ Quercetin/5280343 } & ALA - $26(\mathrm{H}-\mathrm{O})$ & 2.2 & \multirow{3}{*}{3} & \multirow{3}{*}{-6.82} \\
\hline & & HIS - $29(\mathrm{O}-\mathrm{H})$ & 1.8 & & \\
\hline & & ASP - $6(\mathrm{H}-\mathrm{O})$ & 1.7 & & \\
\hline 3. & Daucosterol/5742590 & HIS - $66(\mathrm{H}-\mathrm{O})$ & 1.6 & 1 & -6.64 \\
\hline \multirow{2}{*}{4.} & \multirow{2}{*}{ Baicalein/5281605 } & THR - $3(\mathrm{O}-\mathrm{H})$ & 2.5 & \multirow{2}{*}{2} & \multirow{2}{*}{-6.62} \\
\hline & & HIS - $29(\mathrm{O}-\mathrm{H})$ & 2.2 & & \\
\hline \multirow{2}{*}{5.} & \multirow{2}{*}{ Cosmosin/5280704 } & $\mathrm{ASP}-32(\mathrm{H}-\mathrm{O})$ & 1.9 & \multirow{2}{*}{2} & \multirow{2}{*}{-6.58} \\
\hline & & ASP - $6(\mathrm{H}-\mathrm{O})$ & 1.7 & & \\
\hline \multirow{3}{*}{6.} & \multirow{3}{*}{ Kaempferol/5280863 } & HIS - $29(\mathrm{O}-\mathrm{H})$ & 1.8 & \multirow{3}{*}{3} & \multirow{3}{*}{-6.43} \\
\hline & & ALA - $26(\mathrm{H}-\mathrm{O})$ & 2.1 & & \\
\hline & & ASP - $6(\mathrm{H}-\mathrm{O})$ & 1.7 & & \\
\hline \multirow{3}{*}{7.} & \multirow{3}{*}{ Robinetin/5281692 } & HIS - 29 (H-O) & 1.7 & \multirow{3}{*}{3} & \multirow{3}{*}{-6.4} \\
\hline & & HIS - $29(\mathrm{O}-\mathrm{H})$ & 2.6 & & \\
\hline & & ASP - $6(\mathrm{O}-\mathrm{H})$ & 2.1 & & \\
\hline 8. & Luteolin/5280445 & HIS - 29 (H-O) & 2.2 & 3 & -6.29 \\
\hline
\end{tabular}


V. Sankara Vel and A. Arunprasath, Adv. J. Grad. Res.; Vol. 9, Issue 1, pp: 81-95, January 2021

\begin{tabular}{|c|c|c|c|c|c|}
\hline & & ALA - $26(\mathrm{H}-\mathrm{O})$ & 1.9 & & \\
\hline & & THR - $3(\mathrm{O}-\mathrm{H})$ & 2.4 & & \\
\hline \multirow{2}{*}{9.} & \multirow{2}{*}{ Acacetin/5280442 } & HIS - $29(\mathrm{O}-\mathrm{H})$ & 2.2 & \multirow{2}{*}{2} & \multirow{2}{*}{-5.91} \\
\hline & & GLY - $2(\mathrm{O}-\mathrm{H})$ & 2.6 & & \\
\hline \multirow{2}{*}{10.} & \multirow{2}{*}{ Catechin/9064 } & THR - 3 (H-O) & 1.8 & \multirow{2}{*}{2} & \multirow{2}{*}{-5.76} \\
\hline & & ASP - (H-O) & 1.7 & & \\
\hline \multirow{2}{*}{11.} & \multirow{2}{*}{ Epicatechin/72276 } & ASP - $6(\mathrm{H}-\mathrm{O})$ & 1.7 & \multirow{2}{*}{2} & \multirow{2}{*}{-5.76} \\
\hline & & THR - 3 (H-O) & 1.8 & & \\
\hline \multirow{4}{*}{12.} & \multirow{4}{*}{ Isovitexin/162350 } & ASP - $6(\mathrm{H}-\mathrm{O})$ & 2.1 & \multirow{4}{*}{4} & \multirow{4}{*}{-5.73} \\
\hline & & ASP - $6(\mathrm{H}-\mathrm{O})$ & 2.3 & & \\
\hline & & ASP - $6(\mathrm{H}-\mathrm{O})$ & 1.6 & & \\
\hline & & MET - $1(\mathrm{O}-\mathrm{H})$ & 2.2 & & \\
\hline \multirow{2}{*}{13.} & \multirow{2}{*}{ (+)- Gallacatechin/65084 } & ASP - $6(\mathrm{H}-\mathrm{O})$ & 2.1 & \multirow{2}{*}{2} & \multirow{2}{*}{-5.67} \\
\hline & & THR - 3 (H-O) & 1.9 & & \\
\hline \multirow{2}{*}{14.} & \multirow{2}{*}{ 3-o-Methyl-D-glucose/8973 } & ALA - $7(\mathrm{H}-\mathrm{O})$ & 2.0 & \multirow{2}{*}{2} & \multirow{2}{*}{-5.67} \\
\hline & & ALA - $26(\mathrm{H}-\mathrm{O})$ & 1.8 & & \\
\hline \multirow{2}{*}{15.} & \multirow{2}{*}{ Chanoclavine/5281381 } & ASP - $6(\mathrm{H}-\mathrm{O})$ & 1.7 & \multirow{2}{*}{2} & \multirow{2}{*}{$-5 . .65$} \\
\hline & & ASP - $6(\mathrm{H}-\mathrm{O})$ & 1.3 & & \\
\hline \multirow{3}{*}{16.} & \multirow{3}{*}{ Adenosine/60961 } & THR - 3 (H-O) & 2.0 & & \\
\hline & & ASP - $6(\mathrm{H}-\mathrm{O})$ & 1.7 & 3 & -5.53 \\
\hline & & ASP - $6(\mathrm{H}-\mathrm{O})$ & 2.1 & & \\
\hline 17. & Naringenin/932 & HIS - $29(\mathrm{O}-\mathrm{H})$ & 2.3 & 4 & -5.17 \\
\hline 18. & Daidzein/5281708 & HIS - $29(\mathrm{O}-\mathrm{H})$ & 2.0 & 1 & -51 \\
\hline & & ASP - $6(\mathrm{H}-\mathrm{O})$ & 1.6 & & \\
\hline 19. & Penmmciavme/ 1 524 / & HIS - $29(\mathrm{H}-\mathrm{N})$ & 1.9 & 2 & -4.93 \\
\hline & & ASP - 6 (H-O) & 1.6 & & \\
\hline 20. & Isopenniclavine/12311156 & HIS - $29(\mathrm{H}-\mathrm{N})$ & 1.9 & 2 & -4.93 \\
\hline 21. & Esculetin/5281416 & HIS - $29(\mathrm{O}-\mathrm{H})$ & 2.2 & 1 & -493 \\
\hline 22. & Ciprofloxacin/2764 & GLU - $59(\mathrm{H}-\mathrm{O})$ & 1.9 & 1 & -4.89 \\
\hline & & ASP - $6(\mathrm{H}-\mathrm{O})$ & 1.7 & & \\
\hline 23. & Lysergo1/1498/ & THR - 3 (H-O) & 1.9 & 2 & -4.81 \\
\hline 24. & Pueranin/5281807 & $\begin{array}{c}\text { ASP - } 6(\mathrm{H}-\mathrm{O}) \text { ASP - } \\
6\left(\mathrm{H}_{-} \mathrm{O}\right)\end{array}$ & $\begin{array}{r}2.3 \\
18 \\
\end{array}$ & 2 & -4.86 \\
\hline
\end{tabular}


Evaluation of Fungal Activity Through In Silico Analysis of Medicinal Plants Against Exophiala Jeanselmei

\begin{tabular}{|c|c|c|c|c|c|}
\hline \multirow{2}{*}{25.} & \multirow{2}{*}{ 6-Hydroxy flavone/ 72279} & HIS - 29(O-H) & 2.1 & \multirow{2}{*}{2} & \multirow{2}{*}{-4.81} \\
\hline & & THR - $3(\mathrm{O}-\mathrm{H})$ & 2.5 & & \\
\hline 26. & Teucladiol/16046185 & ALA - $26(\mathrm{H}-\mathrm{O})$ & 2.0 & 1 & -4.8 \\
\hline \multirow{2}{*}{27.} & \multirow{2}{*}{ Ethyl caffetate/ 5317238} & HIS - 29 (O-H) & 2.4 & \multirow{2}{*}{2} & \multirow[b]{2}{*}{-4.78} \\
\hline & & ASP - $6(\mathrm{H}-\mathrm{O})$ & 1.8 & & \\
\hline \multirow{3}{*}{28.} & \multirow{3}{*}{ Buetin/5281222 } & ASP - $5(\mathrm{H}-\mathrm{O})$ & 2.0 & \multirow{3}{*}{3} & \multirow{3}{*}{-4.74} \\
\hline & & ASP - $5(\mathrm{H}-\mathrm{O})$ & 1.9 & & \\
\hline & & ARG - $9(\mathrm{O}-\mathrm{H})$ & 2.1 & & \\
\hline \multirow{2}{*}{29.} & \multirow{2}{*}{ 20-hydroxyecdysone/5459840 } & PHE - 8 (H-O) & 2.0 & \multirow{2}{*}{2} & \multirow{2}{*}{-4.74} \\
\hline & & PHE - 8 (H-O) & 2.3 & & \\
\hline 30. & Rubiadin/ 5124062 & ASP - $6(\mathrm{H}-\mathrm{O})$ & 1.8 & 1 & -4.67 \\
\hline \multirow{3}{*}{31.} & \multirow{3}{*}{ Mimosic acid/190359 } & THR - $3(\mathrm{H}-\mathrm{O})$ & 1.9 & \multirow{3}{*}{3} & \multirow{3}{*}{-4.66} \\
\hline & & $\mathrm{THR}-3(\mathrm{O}-\mathrm{H})$ & 1.6 & & \\
\hline & & $\mathrm{GLY}-2(\mathrm{O}-\mathrm{H})$ & 2.3 & & \\
\hline 32. & Octadecatrienoic acid/57397401 & LYS - $56(\mathrm{O}-\mathrm{H})$ & 2.4 & 1 & -4.46 \\
\hline 33. & Tetracaine/5411 & ALA - $26(\mathrm{H}-\mathrm{O})$ & 2.0 & 1 & -4.25 \\
\hline \multirow{2}{*}{34.} & \multirow{2}{*}{ Chicanine/53360432 } & $\mathrm{ARG}-9(\mathrm{O}-\mathrm{H})$ & 2.1 & \multirow{2}{*}{2} & \multirow{2}{*}{-4.22} \\
\hline & & $\mathrm{ASP}-5(\mathrm{O}-\mathrm{H})$ & 1.8 & & \\
\hline \multirow{2}{*}{35.} & \multirow{2}{*}{ Saupirin/181128 } & $\mathrm{ASP}-6(\mathrm{H}-\mathrm{O})$ & 1.7 & \multirow{2}{*}{2} & \multirow{2}{*}{-4.21} \\
\hline & & ALA - $26(\mathrm{H}-\mathrm{O})$ & 1.8 & & \\
\hline 36. & Elymoclavine/440904 & $\mathrm{ASP}-6(\mathrm{H}-\mathrm{O})$ & 1.6 & 1 & -4.18 \\
\hline \multirow{3}{*}{37.} & \multirow{3}{*}{ Tyrosine/6057 } & $\mathrm{THR}-3(\mathrm{O}-\mathrm{H})$ & 2.3 & \multirow{3}{*}{3} & \multirow{3}{*}{-4.18} \\
\hline & & $\mathrm{ASP}-6(\mathrm{H}-\mathrm{O})$ & 1.8 & & \\
\hline & & ALA - $26(\mathrm{H}-\mathrm{O})$ & 1.9 & & \\
\hline \multirow{3}{*}{38.} & & ALA - $26(\mathrm{H}-\mathrm{O})$ & 2.1 & & \\
\hline & p-coumaric acid/637542 & $\mathrm{THR}-3(\mathrm{O}-\mathrm{H})$ & 2.2 & 3 & -4.13 \\
\hline & & GLY - $2(\mathrm{O}-\mathrm{H})$ & 2.3 & & \\
\hline
\end{tabular}




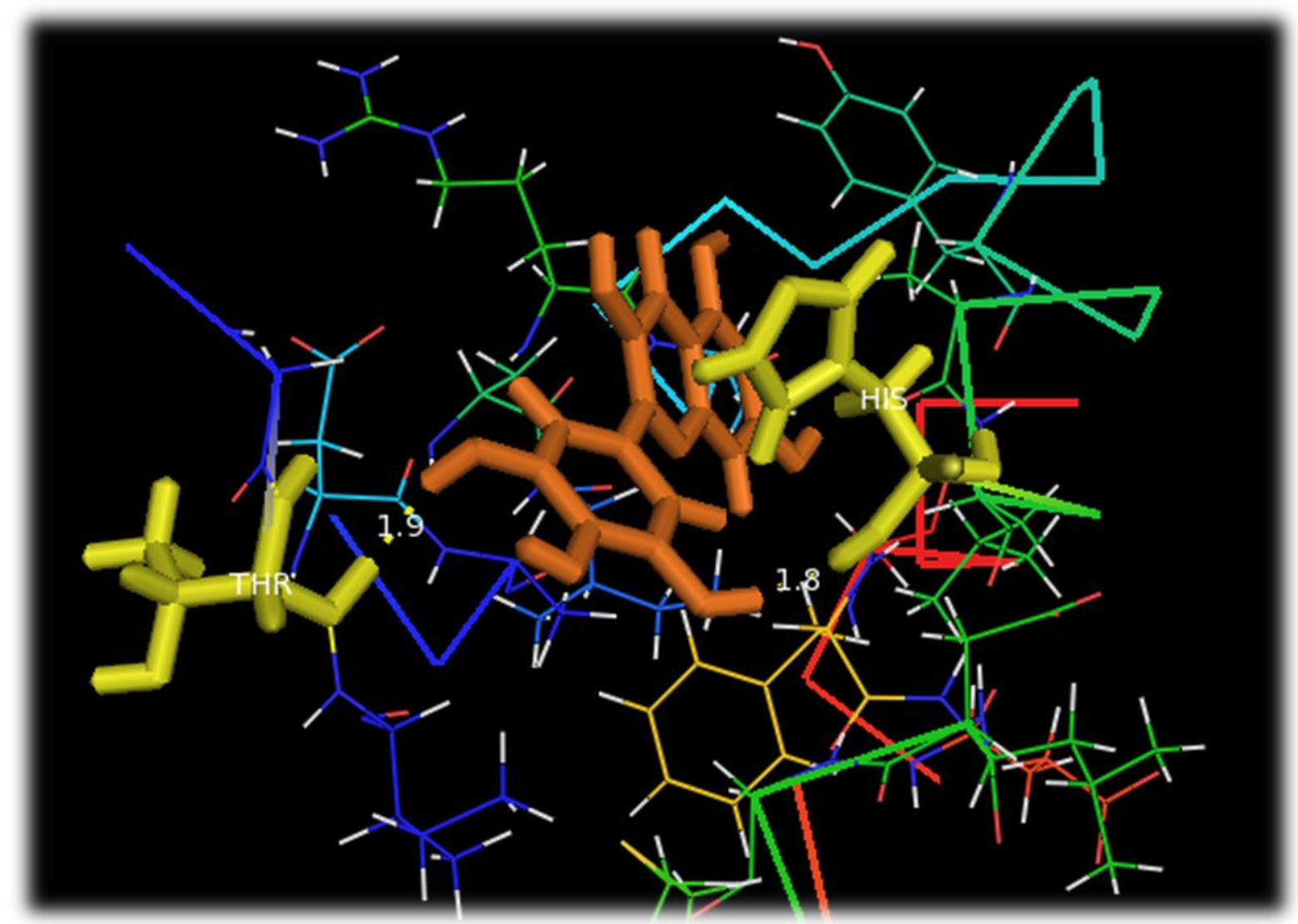

Figure 1: Interaction between 2mpk and Myricetin

The ligand was exposed in TV orange color and interacting residues in yellow color. The dotted lines indicating the hydrogen bond formation. The compound Myricetin had Binding energy of $-7.32 \mathrm{Kcal} / \mathrm{mol}$ and formed hydrogen bonds with the residue HIS 29 showing the bond length of $1.9 \AA$.

\section{Conclusions}

The development of computational methods for protein flexibility is still in its infancy and thereby remains one of the major future directions in protein ligand docking. Rising new contagious species and the occurrence of raised medication obstruction for fungal infections keeps on rising and the height of contagious contaminations is disturbing. Medicinal plants are an important part of our natural health. They serve as important therapeutic agents as well as valuable raw materials for manufacturing numerous traditional and modern medicines. Cassia auriculata leaves have been traditionally used worldwide for its versatile therapeutic properties. A study of molecular docking stimulation undertaken to identify and accesses the binding capacity of ligands. The chemical compounds are used to cure disease and those compounds are found in aerial parts of the plant. The purpose of this study is to analyze the inhibitory potential Chitin synthase I through in silico molecular docking studies on active compounds of plant extract. The protein and ligand docking studies are done by using 3D structure of protein. Most of the molecular docking studies successfully predict the binding modes of small-molecule ligands within receptor binding sites. The phytochemical compounds of Myricetin were isolated from C. auriculata exhibit a good binding efficiency with the target showing high binding energy of $-7.32 \mathrm{Kcal} / \mathrm{mol}$ is used to cure the Phaeohyphomycosis. The biological activity of plant compounds is predicted using pass prediction and the results are proved the chemical compounds have a dermatological property. This indicates that the Myricetin compound has the highest Melanin (main virulent factor) inhibiting activity and also other plant compounds. Those bioactive compounds should be explored more in order to identify an efficient and 
potential drug molecule. As shown in the highlighted case studies, molecular docking has been able to identify promising compounds that might represent future solutions in critical areas of human health.

\section{Declarations}

\subsection{Acknowledgements}

The authors are grateful to acknowledge the PG \& Research Department of Botany, PSG College of Arts \& Science College, Coimbatore for providing requirements during the study.

\subsection{Competing Interests}

The authors declared that they do not have any conflict of interest.

\section{How to Cite this Article:}

V. S.. Vel and A. Arunprasath, "Evaluation of Fungal Activity Through In Silico Analysis of Medicinal Plants Against Exophiala Jeanselmei”, Adv. J. Grad. Res., vol. 9, no. 1, pp. 81-95, Jan. 2021.

\section{References}

[1] N. M. Luscombe, D. Greenbaum, and M. Gerstein, "What is bioinformatics? An introduction and overview," Yearb. Med. Inform., vol. 10, no. 01, pp. 83-100, 2001, doi: 10.1055/s-0038-1638103.

[2] S. L. Lyantagaye, "Current Status and Future Perspectives of Bioinformatics in Tanzania," vol. 39, no. 1, pp. 1-11, 2013.

[3] F. S. Collins, A. Patrinos, E. Jordan, A. Chakravarti, R. Gesteland, and L. R. Walters, "New goals for the U.S. Human Genome Project: 1998-2003," Science (80-.). vol. 282, no. $\quad$ 5389, pp. 682-689, 1998, doi: 10.1126/science.282.5389.682.

[4] D. Kuntz, J. M. Blaney, S. J. Oatley, R. Langridge, and T. E. Ferrin, "A Geometric Approach to Macromolecule-Ligand Interactions," 1982.

[5] L. Z. Benet, C. M. Hosey, O. Ursu, and T. I. Oprea, "BDDCS, the Rule of 5 and drugability," Adv. Drug Deliv. Rev., vol. 101, pp. 89-98, 2016, doi: 10.1016/j.addr.2016.05.007.

[6] P. Kettlewell, M. R. Mcginnis, and G. T. Wilkinson, "Phaeohyphomycosis caused by Exophiala spinifera in two cats," Med. Mycol., vol. 27, no. 4, pp. 257-264, 1989, doi: 10.1080/02681218980000341.

[7] N. Brooijmans and I. D. Kuntz, "Molecular recognition and docking algorithms," Annu. Rev. Biophys. Biomol. Struct., vol. 32, pp. 335-373, 2003, doi: 10.1146/annurev.biophys.32.110601.142532.

[8] J. Wereszczynski and J. A. McCammon, Computational Drug Discovery and Design, ed R Baron. 2012.

[9] E. S. Jacobson, "Pathogenic roles for fungal melanins," Clin. Microbiol. Rev., vol. 13, no. 4, pp. 708-717, 2000, doi: 10.1128/CMR.13.4.708-717.2000.

[10] C. Rajendran, B. K. Khaitan, R. Mittal, M. Ramam, M. Bhardwaj, and K. K. Datta, "Phaeohyphomycosis caused by Exophiala spinifera in India1," Med. Mycol., vol. 41, no. 5, pp. 437-441, Oct. 2003, doi: $\quad$ 10.1080/1369378031000153820.

[11] M. G. Rinaldi, "PHAEOHYPHOMYCOSIS," vol. 14, no. 1, pp. 147-153, 1996.

[12] G. M. Morris, R. Huey, and A. J. Olson, UNIT using AutoDock for ligand-receptor docking, no. SUPPL. 24. 2008.

[13] L. Ajello, "Hyalohyphomycosis and phaeohyphomycosis: Two global disease entities of public health importance," Eur. J. Epidemiol., vol. 2, no. 4, pp. 243-251, 1986, doi: 10.1007/BF00419488.

[14] D. E. Bostock, "Exophiala cat d. e. bostock," vol. 92, pp. 479-482, 1982.

[15] A. Kebriaee-zadeh, “Overview of National Drug Policy of Iran," Iran. J. Pharm. Res. IJPR, pp. 1-2, 2010, doi: 10.22037/ijpr.2010.48.

[16] W. M. Haschek and O. B. Kasali, "A case of cutaneous feline phaeohyphomycosis caused by Phialophora gougerotti," Cornell Vet., vol. 67, no. 4, p. 467—471, Oct. 1977, [Online]. Available: http://europepmc.org/abstract/MED/25087299.

[17] G. Cauwenbergh, "Treatment of Phaeohyphomycosis and Pityriasis Versicolor," in Dimorphic Fungi in Biology and Medicine, H. Vanden Bossche, F. C. Odds, and D. Kerridge, Eds. Boston, MA: Springer US, 1993, pp. 405-411.

[18] Sathyabhama, S. Bhageerathi, and S. Raj, "Mycetoma caused by Exophiala jeanselmei," J. Acad. Clin. Microbiol., vol. 16, no. 2, pp. 94-95, Jul. 2014, doi: 10.4103/0972-1282.144734.

[19] Davidson-Hunt, "Ecological Ethnobotany: Stumbling Toward New Practices and Paradigms," MASA J., vol. 16, no. 1, pp. 1-13, 2000.

[20] Jianling Wang; Laszlo Urban, "The impact of early ADME profiling on drug discovery and,” Drug Discov. World, pp. 73-86, 2004.

[21] M. S. Ali-Shtayeh, R. M.-R. Yaghmour, Y. R. Faidi, K. Salem, and M. A. Al-Nuri, "Ali-Shtayeh1998.Pdf," vol. 60, p. 7, 1998.

[22] A. Allouche, "Software News and Updates Gabedit - A Graphical User Interface for Computational Chemistry Softwares," $J$. Comput. Chem., vol. 32, pp. 174-182, 2012.

[23] M. Tahir ul Qamar, S. M. Alqahtani, M. A. Alamri, and L. L. Chen, "Structural basis of $\quad$ SARS-CoV-2 3CLpro and antiCOVID-19 drug discovery from medicinal plants," J. Pharm. Anal., vol. 10, no. 4, pp. 313-319, 2020, doi: 10.1016/j.jpha.2020.03.009.

[24] F. Lopez-Vallejo et al., "Integrating Virtual Screening and Combinatorial Chemistry for Accelerated Drug Discovery," Comb. Chem. High Throughput Screen., vol. 14, no. 6, pp. 475-487, 2011, doi: 10.2174/138620711795767866.

[25] Y. Liu, Z. Dao, C. Yang, Y. Liu, and C. Long, "Medicinal plants used by Tibetans in Shangri-la, Yunnan, China," J. Ethnobiol. Ethnomed., vol. 5, pp. 1-10, 2009, doi: 10.1186/1746-4269-5-15.

[26] W.L. DeLano. "The PyMOL molecular graphics system”. http://www.Pymol.org. 2002. 
[27] Pauli et al., "Discovery of new inhibitors of mycobacterium tuberculosis InhA enzyme using virtual screening and a 3Dpharmacophore-based approach," J. Chem. Inf. Model., vol. 53, no. 9, pp. 2390-2401, 2013, doi: 10.1021/ci400202t.

[28] M. S. Ali-Shtayeh and S. I. Abu Ghdeib, "Antifungal activity of plant extracts against dermatophytes," Mycoses, vol. 42, no. 11-12, pp. 665-672, 1999, doi: 10.1046/j.1439-0507.1999.00499.x.

[29] M. Negri, T. P. Salci, C. S. Shinobu-Mesquita, I. R. G. Capoci, T. I. E. Svidzinski, and E. S. Kioshima, "Early state research on antifungal natural products," Molecules, vol. 19, no. 3, pp. 2925-2956, 2014, doi: 10.3390/molecules19032925.

[30] R. A. Susidarti, Wahyono, and Yamin, "Isolation and Identification of Antibacterial Compound from the leaves of Muehlenbeckia platyclada Meissn,” Int. J. Adv. Pharm. Sci., vol. 2, no. 1, pp. 40-44, 2011.

[31] D. K. Raja, N. S. Jeganathan, and R. Manavalan, "In vitro antimicrobial activity and phytochemical analysis of Cassia auriculata Linn," Int. Curr. Pharm. J., vol. 2, no. 6, pp. 105-108, 2013, doi: 10.3329/icpj.v2i6.14869.

[32] N. L. Sharma, V. Mahajan, R. C. Sharma, and A. Sharma, "Subcutaneous pheohyphomycosis in India - A case report and review," Int. J. Dermatol., vol. 41, no. 1, pp. 16-20, 2002, doi: 10.1046/j.1365-4362.2002.01337.x.

[33] M. Tahir ul Qamar et al., "Computational screening of medicinal plant phytochemicals to discover potent pan-serotype inhibitors against dengue virus," Sci. Rep., vol. 9, no. 1, pp. 1-16, 2019, doi: 10.1038/s41598-018-38450-1.

[34] Q. Zhang et al., "In silico screening of anti-inflammatory constituents with good drug-like properties from twigs of Cinnamomum cassia based on molecular docking and network pharmacology," Trop. J. Pharm. Res., vol. 18, no. 10, pp. 2125-2131, 2019, doi: 10.4314/tjpr.v18i10.18.

[35] S. Ahmed and A. M. Shohael, "In silico studies of four anthraquinones of senna alata 1. As potential antifungal compounds," Pharmacologyonline, vol. 2, pp. 259-268, 2019.

Publish your books with AIJR publisher-

$\checkmark \quad$ Publish with ISBN and DOI.

$\checkmark$ Publish Thesis/Dissertation as Monograph.

$\checkmark$ Publish Book Monograph.

$\checkmark$ Publish Edited Volume/ Book.

$\checkmark$ Publish Conference Proceedings

$\checkmark \quad$ Retain full copyright of your books.

Submit your manuscript at books.aijr.org
Publish your research article in AIJR journals-

$\checkmark$ Online Submission and Tracking

$\checkmark$ Peer-Reviewed

$\checkmark$ Rapid decision

$\checkmark \quad$ Immediate Publication after acceptance

$\checkmark \quad$ Articles freely available online

$\checkmark$ Retain full copyright of your article.

Submit your article at journals.aijr.org 